\title{
Binary particle separation in droplet microfluidics using acoustophoresis
}

Anna Fornell, Kevin Cushing, Johan Nilsson, and Maria Tenje

Citation: Appl. Phys. Lett. 112, 063701 (2018); doi: 10.1063/1.5020356

View online: https://doi.org/10.1063/1.5020356

View Table of Contents: http://aip.scitation.org/toc/apl/112/6

Published by the American Institute of Physics

\section{Articles you may be interested in}

Graphene bolometer with thermoelectric readout and capacitive coupling to an antenna

Applied Physics Letters 112, 063501 (2018); 10.1063/1.5009629

Guest Editorial: The dawn of gallium oxide microelectronics

Applied Physics Letters 112, 060401 (2018); 10.1063/1.5017845

Spatially dependent carrier dynamics in single InGaN/GaN core-shell microrod by time-resolved cathodoluminescence

Applied Physics Letters 112, 052106 (2018); 10.1063/1.5009728

Particle separation by phase modulated surface acoustic waves

Biomicrofluidics 11, 054115 (2017); 10.1063/1.5001998

Nanoscale quantification of intracellular element concentration by X-ray fluorescence microscopy combined with X-ray phase contrast nanotomography

Applied Physics Letters 112, 053701 (2018); 10.1063/1.5008834

Liquid crystal mediated active nano-plasmonic based on the formation of hybrid plasmonic-photonic modes Applied Physics Letters 112, 061101 (2018); 10.1063/1.5004076

\section{Scilight}

Sharp, quick summaries illuminating the latest physics research

\section{Sign up for FREE!}

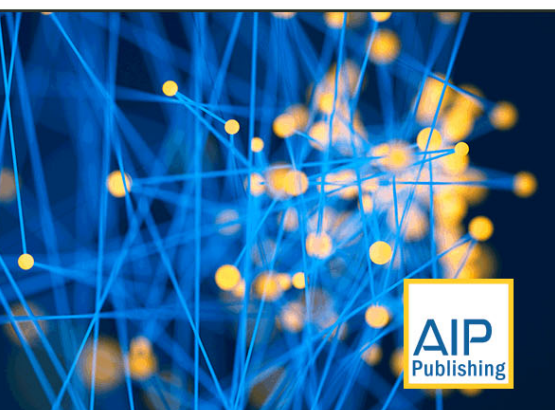




\title{
Binary particle separation in droplet microfluidics using acoustophoresis
}

\author{
Anna Fornell, ${ }^{1, a)}$ Kevin Cushing, ${ }^{1}$ Johan Nilsson, ${ }^{1}$ and Maria Tenje ${ }^{1,2}$ \\ ${ }^{1}$ Department Biomedical Engineering, Lund University, Lund, Sweden \\ ${ }^{2}$ Department Engineering Sciences, Science for Life Laboratory, Uppsala University, Uppsala, Sweden
}

(Received 22 December 2017; accepted 25 January 2018; published online 6 February 2018)

\begin{abstract}
We show a method for separation of two particle species with different acoustic contrasts originally encapsulated in the same droplet in a continuous two-phase system. This was realized by using bulk acoustic standing waves in a $380 \mu \mathrm{m}$ wide silicon-glass microfluidic channel. Polystyrene particles (positive acoustic contrast particles) and in-house synthesized polydimethylsiloxane (PDMS) particles (negative acoustic contrast particles) were encapsulated inside water-in-oil droplets either individually or in a mixture. At acoustic actuation of the system at the fundamental resonance frequency, the polystyrene particles were moved to the center of the droplet (pressure node), while the PDMS particles were moved to the sides of the droplet (pressure anti-nodes). The acoustic particle manipulation step was combined in series with a trifurcation droplet splitter, and as the original droplet passed through the splitter and was divided into three daughter droplets, the polystyrene particles were directed into the center daughter droplet, while the PDMS particles were directed into the two side daughter droplets. The presented method expands the droplet microfluidics tool-box and offers new possibilities to perform binary particle separation in droplet microfluidic systems. Published by AIP Publishing. https://doi.org/10.1063/1.5020356
\end{abstract}

Droplet microfluidics has emerged as a powerful technology for fast and sensitive analytical analysis on-chip. Typically, water-in-oil droplets are generated where one major application of the technology is to use the droplets as individual reaction chambers for bead- and cell-based assays. ${ }^{1,2}$ Monodisperse droplets can be generated at high frequency, and the technology has several other advantages such as fast and controlled reaction times and reduced cost due to the small amount of samples and reagents required for each analysis compared with standard methods. Examples of applications include single-cell analysis, ${ }^{3,4}$ droplet PCR (polymerase chain reaction), ${ }^{5,6}$ and high throughput assays. ${ }^{7,8}$

In many applications, the precise control of the individual droplets and the content inside the droplets is required. ${ }^{9}$ For this purpose, unit-operators to sort, ${ }^{10,11}$ trap, ${ }^{12,13}$ and manipulate particles inside droplets ${ }^{14-20}$ have been developed. Manipulation of particles encapsulated inside droplets is of high interest since it can be used for numerous applications such as particle enrichment and washing. Recently, several methods to control the position of particles inside droplets have been reported including acoustophoresis, ${ }^{14,15}$ dielectrophoresis, ${ }^{16}$ magnetophoresis, ${ }^{17,18}$ and methods relying on sedimentation and the hydrodynamic flow patterns inside the droplets. ${ }^{19,20}$ However, methods to separate one particle species from another encapsulated in the same droplet are still missing. Here, we have developed such a technology by combining an acoustic particle manipulation step with a droplet splitter to separate particles based on the material properties (the acoustic contrast factor).

During the last few years, acoustic particle manipulation (acoustophoresis) has been used to focus, concentrate, and separate particles in various one-phase microfluidic systems, ${ }^{21-23}$ and recently, acoustics has also been implemented

\footnotetext{
${ }^{\text {a) }}$ Author to whom correspondence should be addressed: anna.fornell@ bme.lth.se
}

in two-phase systems to sort whole droplets and to manipulate particles inside droplets. ${ }^{11,14,15,24,25}$ Particles in an acoustic standing wave-field will experience an acoustic radiation force $\left(F_{\mathrm{rad}}\right)$, and the force on a particle in a 1-dimensional $\lambda / 2$-standing wave-field is described by

$$
\begin{gathered}
F^{\mathrm{rad}}=4 \pi \Phi(\tilde{\kappa}, \tilde{\rho}) k a^{3} E_{\mathrm{ac}} \sin (2 k y), \\
\Phi(\tilde{\kappa}, \tilde{\rho})=\frac{1}{3}\left[\frac{5 \tilde{\rho}-2}{2 \tilde{\rho}+1}-\tilde{\kappa}\right], \text { where } \tilde{\rho}=\frac{\rho_{\mathrm{p}}}{\rho_{0}} \text { and } \tilde{\kappa}=\frac{\kappa_{\mathrm{p}}}{\kappa_{0}},
\end{gathered}
$$

where $\Phi$ is the acoustic contrast factor, $k$ is the wavenumber $(k=2 \pi / \lambda), a$ is the radius of the particle, $\lambda$ is the wavelength of the sound, $E_{\mathrm{ac}}$ is the acoustic energy density, $y$ is the distance from the wall, $\rho_{\mathrm{p}}$ and $\rho_{0}$ are the densities of the particle and the fluid, and $\kappa_{p}$ and $\kappa_{0}$ are the compressibilities of the particle and the fluid. ${ }^{26}$ As seen in Eq. (1), the direction of the acoustic force on the particle depends on the sign of the acoustic contrast factor. A particle with positive acoustic contrast is moved to the pressure node, while a particle with negative acoustic contrast is moved to the pressure anti-nodes (Fig. 1). Cells and most common microparticles used in bead-based assays such as polystyrene, glass, and silica particles all have a positive acoustic contrast factor in water. However, there are a few natural occurring examples of negative acoustic contrast particles such as fat particles in

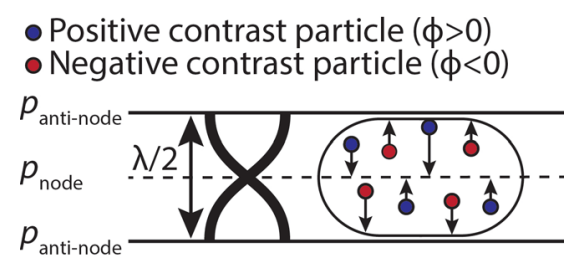

FIG. 1. In an acoustic standing wave-field, microparticles are moved to the pressure node or anti-nodes depending on the acoustic contrast factor. 
(a)

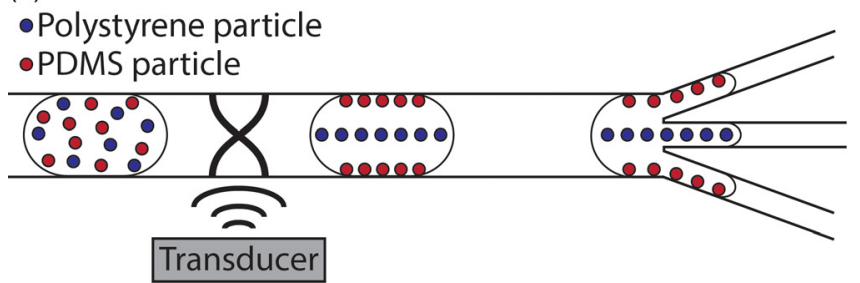

(b)

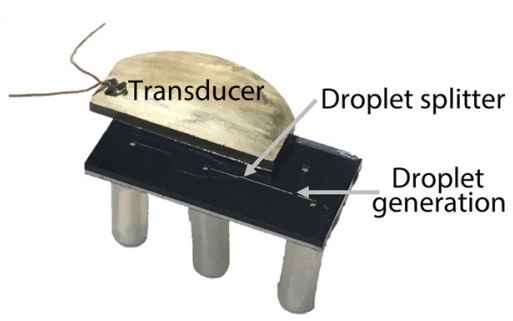

FIG. 2. (a) Schematic of the separation principle. Droplets containing two different particle species are generated, and in the acoustic standing wave-field, the two particle species are separated based on the acoustic contrast. In the droplet splitter, the polystyrene particles, focused in the center of the droplets, are directed into the center daughter droplet, while the PDMS particles, focused to the sides of the droplets, are directed into the side daughter droplets. (b) Photograph showing the fabricated microfluidic chip and the attached piezoelectric transducer. The dimensions of the chip are $2 \mathrm{~cm} \times 1 \mathrm{~cm}$.

milk and lipid particles in human blood. ${ }^{21,27}$ In addition, researchers have synthesized particles in special materials that exhibit negative acoustic contrast in water such as polymershelled gas bubbles ${ }^{28}$ and particles made of PDMS (polydimethylsiloxane). ${ }^{29}$ For our study, standard polystyrene particles were used as positive acoustic contrast particles and PDMS particles were synthesized and used as negative acoustic contrast particles. PDMS particles were used as negative acoustic contrast particles since in future applications, these particles can be easily surface-functionalized to enable separation of bioparticles. ${ }^{30}$ However, surface-functionalizing of PDMS particles is outside the scope of this paper where we show the proof-of-principle of separation of two particle species inside droplets.

The microfluidic system is shown in Figs. 2(a) and 2(b). The channels were etched on a silicon wafer using deep reactive-ion etching and sealed with a glass lid using anodic bonding. The height of all channels was $100 \mu \mathrm{m}$, and the main channel was $380 \mu \mathrm{m}$ wide which gives a $\lambda / 2$-resonance close to $2 \mathrm{MHz}$. The droplet splitter consisted of a trifurcation where the side outlet channels were angled $20^{\circ}$ with respect to the main channel, and the width of these channels was $117 \mu \mathrm{m}$. The channels were hydrophobic surface-coated using silane (Repel-Silane ES, GE Healthcare). A $1 \mathrm{~mm}$ thick piezoelectric transducer (PZT26, Ferroperm or APC840, APC International) having fundamental resonance at $2 \mathrm{MHz}$ was glued to the chip.

The transducer was actuated by an AC-signal, and the voltage over the transducer was $22 \mathrm{~V}_{\text {peak-peak }}$ and the frequency was $1.83-1.85 \mathrm{MHz}$. To generate droplets, the incoming fluid phases were controlled by two syringe pumps (NEMESYS, Cetoni) operated in the continuous injection mode. The continuous phase was olive oil (Di Luca \& Di Luca), and the disperse phase was water containing microparticles (polystyrene particles and/or PDMS particles).

To control the droplet splitting, the flow rates in the side outlets were set by one common syringe pump (NEMESYS, Cetoni) operated in the withdrawal mode while the center channel outlet was connected directly to an open container. The total flow rate was $2-3 \mu \mathrm{l} / \mathrm{min}$ in all experiments except for the video in the supplementary material. Images of the experiments were acquired using a camera (XM10, Olympus) mounted on an optical microscope (BX51W1, Olympus).

The key feature in this work is separation of two particle species into individual daughter droplets based on the material properties of the particles. To demonstrate this concept, polystyrene particles (10 $\mu$ m diameter, Sigma-Aldrich) were used along with PDMS particles. The PDMS particles were prepared according to a similar protocol as described by Choi et al. ${ }^{31}$ First, PDMS (Sylgard 184, Dow Corning) was mixed in a 10:1 ratio and then degassed. Two plastic syringes $(5 \mathrm{ml}$, Henke Saas Wolf) with syringe needles (18 G blunt fill needle, Becton Dickinson) were connected to each other via a piece of silicone tubing (228-0701, VWR). One of the syringes was filled with $4 \mathrm{ml}$ water and the other syringe was filled with $0.4 \mathrm{ml}$ PDMS. The pistons were manually pushed back-and-forth 5 times to create a PDMS-water emulsion. The resulting emulsion was then ejected into a bottle with $10 \mathrm{ml}$ water placed on a heating plate $\left(74^{\circ} \mathrm{C}\right)$ under constant stirring. To ensure that all PDMS particles had cross-linked completely, the bottle was kept on the heating plate for $1 \mathrm{~h}$. In Fig. 3, a photograph of the PDMS particles is shown, and as seen in the photograph, the synthesized PDMS particles are highly spherical and have a wide size distribution. In applications where a narrow particle size distribution is wanted, size-fractionation using, for example, centrifugation can be employed. ${ }^{30}$ However, in this study, we wanted to evaluate the acoustophoretic manipulation of a large range of particle sizes, and thus, no size-fractionation was performed.

To characterize the system, water droplets containing polystyrene particles and/or PDMS particles were generated. In the first experiment, only polystyrene particles were encapsulated inside the droplets. Without the ultrasound, the polystyrene particles were positioned in the entire droplet [Fig. 4(a)], and when the ultrasound was applied at the fundamental resonance frequency, the polystyrene particles were focused to the center of the droplet [Fig. 4(b)]. In the second experiment, PDMS particles were encapsulated



FIG. 3. Photograph of the synthesized PDMS particles. 
(a) Sound off: Polystyrene particles

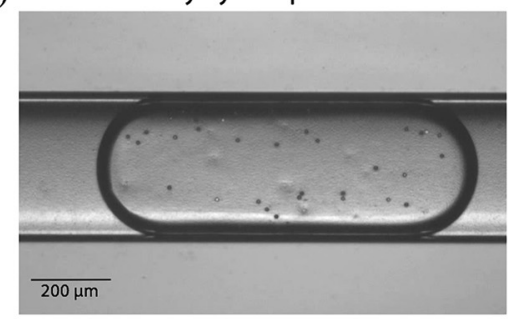

(b) Sound on: Polystyrene particles

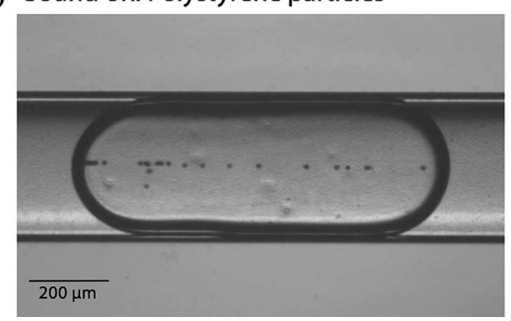

(c) Sound off: PDMS particles

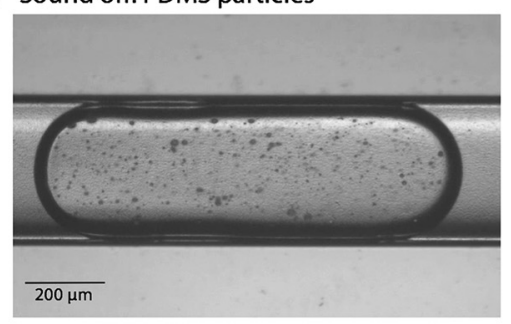

(d) Sound on: PDMS particles

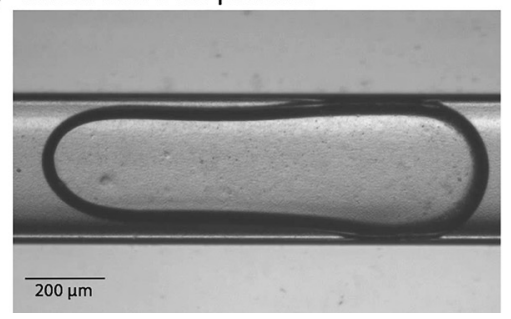

(e) Sound off: Polystyrene and PDMS particles

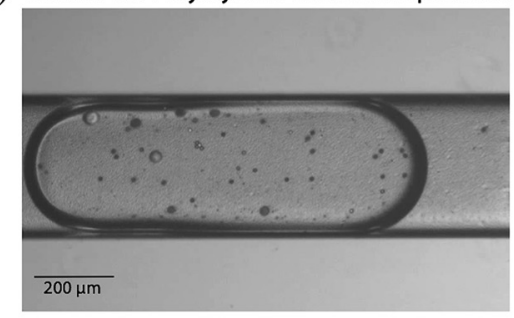

(f) Sound on: Polystyrene and PDMS particles

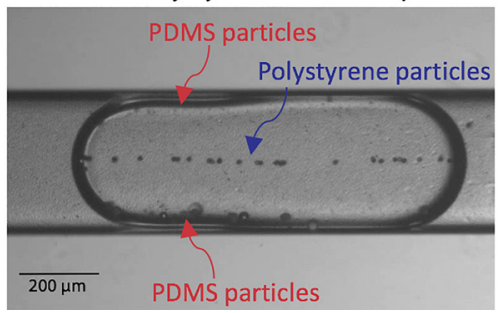

FIG. 4. Without the ultrasound applied, the polystyrene particles (a), PDMS particles (c), and a mixture of these (e) are all positioned throughout the entire droplets. With the ultrasound applied, the polystyrene particles are moved to the center (b), the PDMS particles are moved to the sides (d), and with a mixture of the two particle species, the polystyrene particles are moved to the center, while the PDMS particles are moved to the sides (f). The direction of flow is towards the right in the images.

instead. Without the ultrasound, the PDMS particles were positioned in the entire droplet [Fig. 4(c)], and when the ultrasound was applied at the fundamental resonance frequency, the PDMS particles were moved towards the sides of the droplet [Fig. 4(d)]. As seen in Fig. 4(d), both the smaller and larger PDMS particles could be moved by the ultrasound. In the supplementary material, a video of the movement of the PDMS particles in response to the onset of the ultrasound is available. As seen in Fig. 4(d) and in the video, when the PDMS particles have moved to the sides of the droplet, the PDMS particles are difficult to see due to the dark ring around the droplet. This dark ring arises as a result of the different refractive indices of water and olive oil. ${ }^{32}$ In the third experiment, a mixture of polystyrene particles and PDMS particles was encapsulated inside the droplets. Without the ultrasound, both particles species were positioned in the entire droplet [Fig. 4(e)], and when the ultrasound was applied at the fundamental resonance frequency, the polystyrene particles were focused to the center of the droplet, whereas the PDMS particles were moved towards the sides of the droplet [Fig. 4(f)]. This set of experiments demonstrates that particles encapsulated inside droplets can be moved to the pressure nodes or anti-nodes depending on the acoustic contrast of the particles.

To explore the technology for isolation of two particle species originally encapsulated in the same droplet, a trifurcation droplet splitter was implemented. Droplets containing a mixture of polystyrene particles and PDMS particles were generated, and at the trifurcation, each droplet was divided into three daughter droplets. Without the ultrasound, the polystyrene particles and the PDMS particles were visually found in all three daughter droplets [Fig. 5(a)], and when the ultrasound was applied at the fundamental resonance frequency, the polystyrene particles were focused in the center of the droplet and directed into the center daughter droplet, while the PDMS particles were directed into the side daughter droplets [Fig. 5(b)]. In the supplementary material, a video of the droplet splitting and particle separation is available.
These results demonstrate that acoustophoresis is a suitable technology for binary particle separation in droplet microfluidic systems. During operation of the system, internal fluid motions and vortices were observed in the droplets both with and without the ultrasound applied,,$^{33,34}$ and in order to focus the particles, the acoustic force needs to be stronger than the hydrodynamic force. Overcoming the hydrodynamic force is one of the main challenges in almost all applications where particles are manipulated inside

(a) Sound off: Polystyrene and PDMS particles

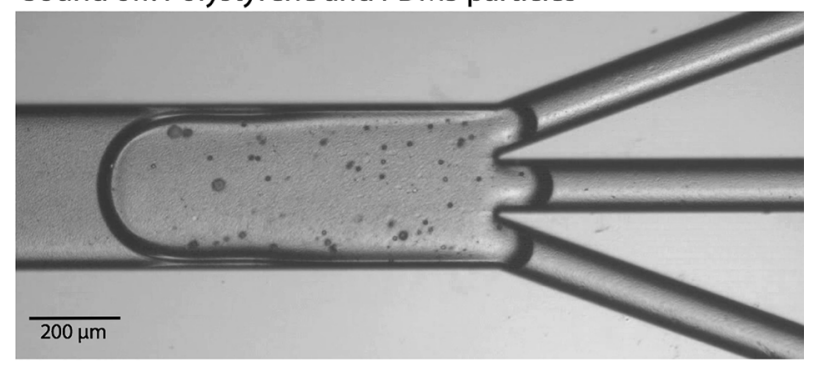

(b) Sound on: Polystyrene and PDMS particles

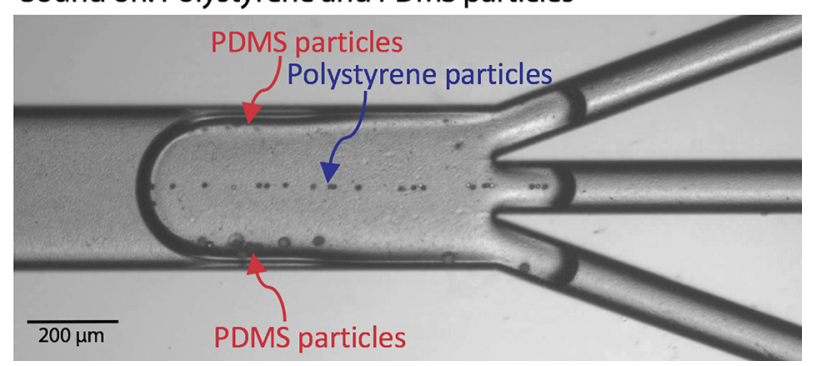

FIG. 5. A mixture of polystyrene particles and PDMS particles is encapsulated, and in the droplet splitter, each droplet is divided into three daughter droplets. Without the ultrasound applied, the two particle species are mixed in both the center and the side daughter droplets (a), while with the ultrasound applied, the polystyrene particles are directed into the center daughter droplet and the PDMS particles are directed into the side daughter droplets. The direction of flow is towards the right in the images. 
droplets using external forces and may limit the throughput of the system. Compared with other particle manipulation methods, the presented method has the advantage of being label-free and generic as the only requirement is that the particles that are to be separated have different acoustic contrasts. Future work includes surface-functionalizing the particles to bind specific cells and biomolecules and combining the acoustic separation unit with other droplet unitoperators to be able to perform complex assays in droplets.

See supplementary material for a video of the movement of the PDMS particles in response to the onset of the ultrasound and a video showing droplet splitting and particle separation.

The authors would like to thank Mathias Ohlin, Klara Björnander Rahimi, Fredrik Ekström, Anders Holmberg, and Karolina Svensson (Uppsala University) for the microfabrication work. This work was funded by the Swedish Research Council, the Crafoord Foundation, the Royal Physiographic Society of Lund, and the Foundation Olle Engkvist Byggmästare.

${ }^{1}$ T. Schneider, J. Kreutz, and D. T. Chiu, Anal. Chem. 85, 3476 (2013).

${ }^{2}$ I. S. Casadevall and A. DeMello, Chem. Commun. 47, 1936 (2011).

${ }^{3}$ J. F. Edd, D. Di Carlo, K. J. Humphry, S. Köster, D. Irimia, D. A. Weitz, and M. Toner, Lab Chip 8, 1262 (2008).

${ }^{4}$ A. M. Klein, L. Mazutis, I. Akartuna, N. Tallapragada, A. Veres, V. Li, L. Peshkin, D. A. Weitz, and M. W. Kirschner, Cell 161, 1187 (2015).

${ }^{5}$ N. R. Beer, E. K. Wheeler, L. Lee-Houghton, N. Watkins, S. Nasarabadi, N. Hebert, P. Leung, D. W. Arnold, C. G. Bailey, and B. W. Colston, Anal. Chem. 80, 1854 (2008).

${ }^{6}$ D. Pekin, Y. Skhiri, J.-C. Baret, D. Le Corre, L. Mazutis, C. B. Salem, F. Millot, A. El Harrak, J. B. Hutchison, J. W. Larson, D. R. Link, P. Laurent-Puig, A. D. Griffiths, and V. Taly, Lab Chip 11, 2156 (2011).

${ }^{7}$ J. J. Agresti, E. Antipov, A. R. Abate, K. Ahn, A. C. Rowat, J.-C. Baret, M. Marquez, A. M. Klibanov, A. D. Griffiths, and D. A. Weitz, Proc. Natl. Acad. Sci. U.S.A. 107, 4004 (2010).

${ }^{8}$ S. L. Sjostrom, Y. Bai, M. Huang, Z. Liu, J. Nielsen, H. N. Joensson, and H. A. Svahn, Lab Chip 14, 806 (2014).
${ }^{9}$ M. Tenje, A. Fornell, M. Ohlin, and J. Nilsson, Anal. Chem. (to be published).

${ }^{10}$ J. C. Baret, O. J. Miller, V. Taly, M. Ryckelynck, A. El-Harrak, L. Frenz, C. Rick, M. L. Samuels, J. B. Hutchison, J. J. Agresti, D. R. Link, D. A. Weitz, and A. D. Griffiths, Lab Chip 9, 1850 (2009).

${ }^{11}$ T. Franke, A. R. Abate, D. Weitz, and A. Wixforth, Lab Chip 9, 2625 (2009).

${ }^{12}$ A. Huebner, D. Bratton, G. Whyte, M. Yang, A. J. DeMello, C. Abell, and F. Hollfelder, Lab Chip 9, 692 (2009).

${ }^{13}$ J. H. Jung, G. Destgeer, J. Park, H. Ahmed, K. Park, and H. J. Sung, Anal. Chem. 89, 2211 (2017).

${ }^{14}$ A. Fornell, J. Nilsson, L. Jonsson, P. K. P. Rajeswari, H. N. Joensson, and M. Tenje, Anal. Chem. 87, 10521 (2015).

${ }^{15}$ A. Fornell, M. Ohlin, F. Garofalo, J. Nilsson, and M. Tenje, Biomicrofluidics 11, 31101 (2017).

${ }^{16}$ S. Han, H. S. Kim, and A. Han, Biosens. Bioelectron. 97, 41 (2017).

${ }^{17}$ E. Brouzes, M. Medkova, N. Savenelli, D. Marran, M. Twardowski, J. B. Hutchison, J. M. Rothberg, D. R. Link, N. Perrimon, and M. L. Samuels, Proc. Natl. Acad. Sci. U.S.A. 106, 14195 (2009).

${ }^{18}$ R. Gao, Z. Cheng, A. deMello, and J. Choo, Lab Chip 16, 1022 (2016).

${ }^{19}$ G. K. Kurup and A. S. Basu, Biomicrofluidics 6, 22008 (2012).

${ }^{20}$ M. Hein, M. Moskopp, and R. Seemann, Lab Chip 15, 2879 (2015).

${ }^{21}$ F. Petersson, A. Nilsson, C. Holm, H. Jonsson, and T. Laurell, Lab Chip 5, 20 (2005).

${ }^{22}$ J. Shi, H. Huang, Z. Stratton, Y. Huang, and T. J. Huang, Lab Chip 9, 3354 (2009).

${ }^{23}$ P. Augustsson, C. Magnusson, M. Nordin, H. Lilja, and T. Laurell, Anal. Chem. 84, 7954 (2012).

${ }^{24}$ I. Leibacher, P. Reichert, and J. Dual, Lab Chip 15, 2896 (2015).

${ }^{25}$ P. R. Rogers, J. R. Friend, and L. Y. Yeo, Lab Chip 10, 2979 (2010).

${ }^{26}$ H. Bruus, Lab Chip 12, 1014 (2012).

${ }^{27}$ C. Grenvall, P. Augustsson, J. R. Folkenberg, and T. Laurell, Anal. Chem. 81, 6195 (2009).

${ }^{28}$ S. V. V. N. Kothapalli, M. Wiklund, B. Janerot-Sjoberg, G. Paradossi, and D. Grishenkov, Ultrasonics 70, 275 (2016).

${ }^{29}$ K. W. Cushing, M. E. Piyasena, N. J. Carroll, G. C. Maestas, B. A. López, B. S. Edwards, S. W. Graves, and G. P. López, Anal. Chem. 85, 2208 (2013).

${ }^{30}$ K. Cushing, E. Undvall, Y. Ceder, H. Lilja, and T. Laurell, Anal. Chim. Acta 1000, 256 (2018).

${ }^{31}$ Y. H. Choi, K. H. Chung, H. B. Hong, and W. S. Lee, Int. J. Polym. Mater. Polym. Biomater. 66, 1 (2017).

${ }^{32}$ O. Carrier, F. G. Ergin, H.-Z. Li, B. B. Watz, and D. Funfschilling, J. Micromech. Microeng. 25, 84014 (2015).

${ }^{33}$ H. Kinoshita, S. Kaneda, T. Fujii, and M. Oshima, Lab Chip 7, 338 (2007).

${ }^{34}$ M. Ohlin, A. Fornell, H. Bruus, and M. Tenje, J. Micromech. Microeng. 27, 84002 (2017). 